\title{
Relationships, associations, risk factors and correlations: nebulous phrases without obvious clinical implications
}

\author{
Lisa A. Harvey ${ }^{1}$ \\ (c) International Spinal Cord Society 2020
}

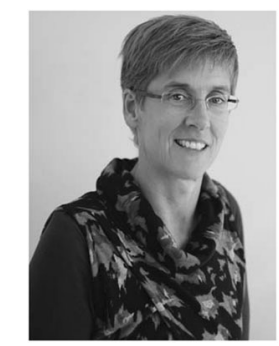

Are you ever left wondering about the purpose or clinical implications of studies in which the aim is "to explore relationships", "investigate associations”, “look at risk factors", or "examine correlations"?

At one level these aims seem reasonable enough. They are certainly very common. Why not explore associations, correlations, relationships and risk factors? There is no shortage of fancy statistical methods to do any of these things, and large database are very conducive to these sorts of analyses. So what is the problem?

The problem is that there is nothing intrinsically meaningful about the relationship or association between variables. Demonstration of the existence of associations and/or the magnitude of any association does not on its own answer a clinical question or test a research hypothesis, and the information can not guide clinical practice or future research. Knowledge of relationships, associations, risk factors or correlations is ultimately only useful for one of two purposes: to predict an outcome or to identify causal mechanisms [1, 2]. Therefore, studies of associations need to make it clear whether their ultimate aim is to predict specified outcomes or identify postulated causal effects. Importantly, the design of the study needs to match the stated aim. Studies that aim to demonstrate causal effects should be designed and analysed very differently from studies that aim to predict outcomes.

Researchers who are interested in predicting outcomes need to identify candidate predictors [3]. Predictors do not need to be on the causal pathway. For example, yellow fingers might be a good predictor of lung cancer. The yellow fingers do not cause the lung cancer. They are merely a predictor of lung cancer-because cigarette smokers may

\footnotetext{
$\triangle$ Lisa A. Harvey

spinalcord@iscos.org.uk

1 University of Sydney, Sydney, Australia
}

get yellow fingers, and smokers may get cancer. A common mistake is to infer that a predictor (sometimes unhelpfully called a risk factor) causes an outcome when the study has not been designed to determine causation (see below paragraphs). This leads to claims about the need to intervene on modifiable predictors to change outcomes. That is obviously inappropriate, as this example shows. Washing fingers to remove the yellow stain will not reduce the risk of lung cancer. Only if evidence can be provided that the predictor causes the outcome is it reasonable to suggest that therapeutic efforts should be directed at this predictive factor.

Predictors must be easy to measure if they are going to be useful for forecasting outcomes in clinical practice. For example, predicting whether a person with recent spinal cord injury will walk one year after injury, based on a few quick and easy clinical tests taken at the time of injury, is going to be far more useful than forecasting ability to walk based on a complex analysis of MRI images. The incremental predictive value of any complex or expensive test, over simpler and cheaper ones, needs to be demonstrated before those complicated and pricy tests can be justified. Importantly, predictors need to be measured at the time the prediction is to be made, and prior to the time the outcome is measured. These two points of time need to be clearly articulated. Data collected after the time at which a prediction is to be made should not be used as candidate predictors. For example, if a prediction model is to be used at the time of injury then it is not meaningful to use amount of rehabilitation provided over the first 6 months after injury to predict walking ability at one year, because at the time of injury it is not known with any certainty how much rehabilitation a person will receive.

What about causation? Everyone learns that association is not causation, and that it is impossible to prove the existence of causal effects using observational data. This understanding has led researchers to avoid saying that they are interested in "causation" [4]. Instead, they use nebulous phrases to refer to the aims of their studies. They say they 
will examine associations, or relationships, or risk factors, or something similar. These terms are obscurantist. They should not be used to avoid an explicit statement of the objective of the research. If the aim is not simply prediction, then it must ultimately be to understand causation. Therefore, the key lesson we all needed to learn at the beginning of our statistical training was not that association does not equal causation, but rather that there is no inherent value in knowing associations unless we are interested in causation or prediction, and an association is only interesting if it is predictive or causal.

Some authors claim that, while they may ultimately be interested in understanding causation, their immediate aim is exploratory data mining to identify associations, which subsequently might be shown to be causal. If that is really the case, this needs to be very clearly stated. However, such work is of low publication priority for Spinal Cord, both because it is often obvious which variables are associated with outcomes (there is no shortage of candidate causes) and because demonstration of associations is intrinsically of no interest. In addition, this type of analysis will have a high type I error rate which will lead to spurious findings.

Of course it is difficult to prove causation. In recent decades theoreticians have shown that the common practice of putting all measured variables into a multivariate statistical model and obtaining adjusted estimates of their effects is theoretically problematic. There is a risk of adjusting for mediators (variables that lie on the causal pathway between the exposure and the outcome) or colliders (variables that are independently caused by both the exposure and the outcome [5]), which creates, rather than reduces, bias in estimates of causal effects. Instead, the process of exploring causal effects needs to start with the construction of a Directed Acyclic Graph (DAG) [6]. DAGs are a graphical way of explicitly stating the theoretical framework that should underlie the estimation of any causal effect. Software such as the freely available DAGitty can be used to create DAGs and to identify sufficient sets of variables that must be controlled for if estimates of causal effects are to be obtained (see recent editorial on a related topic [7]). Spinal
Cord welcomes papers that provide these types of frameworks on important causal questions because if the framework can be agreed upon a priori by the scientific community, then they can be used to guide future observational studies.

Spinal Cord will continue to encourage authors to carefully think about the real underlying purpose and practical value of examining relationships, associations, risk factors and correlations. Authors of studies which examine relationships, associations, risk factors and correlations will be asked to explicitly state if their intention is prediction or understanding causal mechanisms. Studies that do not explicitly articulate one of these aims, or that do not use a strong design for answering questions about prediction or causation, will be considered a low publication priority. Careful consideration of all these important epidemiological research principles will go some way to ensuring that the research published in Spinal Cord is both trustworthy and useful.

\section{References}

1. Schooling CM, Jones HE. Clarifying questions about "risk factors": predictors versus explanation. Emerg Themes Epidemiol. 2018;15:10.

2. Herbert RD. Cohort studies of aetiology and prognosis: they're different. J Physiother. 2014;60:241-4.

3. Moons KG, Royston P, Vergouwe Y, Grobbee DE, Altman DG. Prognosis and prognostic research: what, why, and how? BMJ. 2009;338:b375.

4. Hernan MA. The C-Word: scientific euphemisms do not improve causal inference from observational data. Am J Public Health. 2018;108:616-9.

5. Lee H, Aronson J, Nunan D. Collidor bias. A distortion that modifies an association between an exposure and outcomes, caused by attempts to control for a common effect of the exposure and outcome. Catalogue of bias collaboration; 2019. https://cata logofbias.org/biases/collider-bias/ Accessed 26 Nov 2019.

6. Shrier I, Platt RW. Reducing bias through directed acyclic graphs. BMC Med Res Methodol. 2008;8:70.

7. Chamberlain JD, Brinkhof MWG. Using strong inference to answer causal questions in spinal cord injury research. Spinal Cord. 2019;57:907-8. 